\title{
The Research on Teacher Training Strategies Based on Activation Memory
}

\author{
Long Zhang ${ }^{1,2, *}$, Ramir S. Austria ${ }^{1}$, Jie Tang ${ }^{1,2}$ \\ ${ }^{1}$ University of the Cordilleras, Baguio City, Philippines 2600 \\ ${ }^{2}$ Department of Scientific Research, Liupanshui Normal University, Liupanshui China 553004 \\ *Corresponding author. Email: lpslong@139.com
}

\begin{abstract}
With the rapid development of brain and cognitive neuroscience, the research results of brain science are applied to teacher training. By inducing the long-lasting semantic memory, creating the deep episodic memory, optimizing the efficient procedural memory, stimulating the associative automatic memory and mobilizing the positive emotional memory, the memory routes are opened, and the smooth memory retrieval is realized, so as to achieve the training significance.
\end{abstract}

Keywords: Brain science, memory, teacher training, memory retrieval

\section{INTRODUCTION}

In recent years, with the rapid development of brain and cognitive neuroscience, brain science has become one of the most cutting-edge, important and active disciplines in the current scientific research field. Brain science is used in various industries, and among them, brain science application and teacher training make teachers no longer carry out educational practice based on intuition but start to pay attention to the reality and rationality of their own actions. However, the biggest drawback of intuitive practice is that the content of intuition cannot be effectively transmitted, and the practical skills of intuition cannot often be taught to others. In order to make up for its drawbacks, the language, learning, memory, thinking, emotion, social behavior and other advanced brain functions are regarded as the important contents of the research on cognitive neuroscience, and the great influence of brain plasticity on the process and results of teacher training is concerned. Teacher training is conducted according to the laws of brain development and activity and the laws of brain's cognitive activity. On the premise of fully understanding the brain's cognitive functions, emotional functions, self-consciousness and other advanced functions, it is a scientific and effective training strategy to carry out the training that adapts to the development characteristics of adult cognitive ability.

\section{TRAINING AND MEMORY}

Working memory is the coordination of various brain systems through the role of attention to complete the functions of perception, representation and action planning [1]. Ranganath $\mathrm{C}$ and other researchers have studied working memory based on the activation model, believing that working memory activation is the temporary activation of long-term memory representation, and the activation of long-term memory representation contains all the information in working memory [2]. The memory in training is actually the ability to memorize some common sense, knowledge and new ideas or repeat some actions, so as to achieve the infinite reproduction and extension of excellent results for teachers' future educational practices. The most critical and fundamental thing in the implementation of teacher training is to pay attention to the quality and efficiency of teacher training, so as to achieve the training goal of efficient teachers of improving teachers' knowledge and skill reserve.

\subsection{The Relationship between Teachers' Learning and Memory}

Human beings can already observe the function of the biological human brain and can closely link learning, education and brain science, so that learning and education can be regarded as the concepts that summarize the entire life from life to death to redefine them in the biological sense: learning is the process of constructing the central neural network through external environmental stimuli, while education is the process of controlling and improving external stimuli [3]. In the school system education from primary school to university, teachers should first learn and understand knowledge, and then acquire educational experience through field training and have judgment and creativity. Finally, they should have the comprehensive ability of educational wisdom. "Brain cells are far more interconnected than the Internet." Teacher training must train the brain as well, because the more the brain is used, the more active the brain is. As teachers participate in new training, their brains will continue to grow into new branches. Without training and learning, teachers will slowly lose the important network parts that affect their memory, learning ability and motivation. 
Therefore, brain science has improved teachers' ability to adapt to the times of rapid change.

\subsection{The Relationship between Teachers' Skill Development and Memory}

The psychological mechanism of the formation process of teachers' educational and teaching skills is perception, cognition and thinking. The core of the realization of these three processes is memory. On the basis of memory, the will is formed, which is expressed by self-consciousness and behavior, and finally, decisions, plans and actions are made[4]. The human brain not only recognizes patterns, but also looks for patterns, and even produces patterns where they don't exist. Relying on training to develop teachers' good memory provides the physiological premise for finding and establishing stable skill patterns and innovating new skill patterns.

\subsection{The Relationship between Teacher Training Effect and Memory}

The human brain does not store memory in a linear way like a recorder or video recorder, but stores the contents of the memory in neural circuits or neural networks. Effective training must be able to attract all the feelings of teachers. It is necessary to use seeing, hearing, doing and practice to consciously create memories, activate previously established neural circuits or networks that store relevant information and reconstruct and reproduce previous information. If the brain can't find the old network that was activated to match the new information, the brain is likely not to notice it. In general, information that is not identified as important cannot be remembered. All people, including teachers, cannot survive by memorizing and storing meaningless information [5]. If the information is processed more fully, more neural connections will be formed, and the memory will be more consolidated, so the memory effect will be better [6]. Training is a social activity, and teachers' subjective participation influences the effect, because the joint activities between the team and teachers in the training may promote the brain to release positive chemicals. The purpose of teacher training is to implement constructive promotion work in the face of the comprehensive quality of teachers, not to seek a single and correct "way of doing things". The trainer imposes a style on teachers, which is equivalent to imposing a wrong self on others. It is necessary to activate and awaken the memory, connect and recombine the training content with teachers' existing cognitive structure and form the understanding of teachers' individual characteristics, so as to obtain the successful strategies of teacher training. For each teacher, training activities and peers can play a certain role in creating the rich environment. Teachers' active participation in training activities can promote brain activity and enhance memory. However, too much pressure will hinder the work of the brain, ossify the connection of practice blocks, limit the immediate retrieval of memory content and gradually fade away teachers' personalized style. Practice has proved that positive feedback is the most obvious way to affect brain chemicals [7], and good self-perception and job satisfaction contribute to the production of positive chemicals in the body, while the stimulation and challenge to brain promote the growth of brain and prolong the professional life of teachers.

\section{TRAINING SKILLS ACTIVATE MEMORY ROUTES}

For teachers in training, if they want to remember the past or the future knowledge or behavior, they must follow a certain path, namely "route". In the study of activation memory, Cowan and Oberauer have advocated long-term memory, and Cowan has believed that attention processing, short-term memory and long-term memory are contained in layers [8], while Oberauer [9] has argued that working memory system consists of three parts, which are (in a narrow sense) attention focus, direct access area and activation area of long-term memory [10]. The brain filters from all incoming sensory stimuli, choosing the most relevant and meaningful information to focus on. The brain is determining which information is meaningful and which information is irrelevant in the initial process of perceptual processing and in the process of conscious information processing. Therefore, we can properly compare the brain's information storage mechanism to the joint network. Information suitable or able to be added to the existing network is naturally more likely to be stored than information without these attributes [11]. The following five memory routes have their own entrances for access to information. If the right one is followed, you can lead to the information stored in long-term memory, which lays the information foundation for the next teachers' practice. Thus, during teacher training, the trainer must properly use various training skills, process the information in the attention focus and activate some passive dormancy waiting information of long-term memory information. Only through training, some past knowledge can be further activated, and teachers' memory routes can be opened.

\subsection{Inducing the Long-Lasting Semantic Memory}

In the process of teacher training, the active level of memory is affected by external stimuli, such as environment (light, sound, temperature and layout), society (trainer, peer and self), emotion (structure, persistence, motivation and responsibility), body (perceptual intensity: hearing, vision and touch) and psychology [12]. Matching teachers' preferences with training situations will improve training behavior and effect. Relatively speaking, the impact of auditory stimuli on teachers is the most frequent. Semantic memory is to keep information from speech 
based on the auditory route, and the environment of many teacher training activities depends on semantic memory. It is not an easy process for teachers to receive training by using the semantic memory route. Teachers' brains need to classify and review all old and new information through the repeated processing and the stimulation of association, contrast and analogy, and then new and old information are linked to form long-term memory.

\subsection{Creating the Deep Episodic Memory}

The brain is very sensitive to environmental stimuli and effect and produces episodic memory. Compared with semantic memory, it is much easier to enter the episodic memory route. The key part of this memory route is that when you are learning something, you must be in a certain position, so you can easily connect the learning process with the location. Training relies on information processing, cognitive skills and basic habits of using memory, and the training effect is the product of the interaction between cognition (wisdom) and situation (task). Due to the existence of CMT, episodic memory is easily polluted, because teachers have a lot of experience, and confusion is inevitable. This kind of memory can help teachers improve their practical skills through experience, which is also necessary for teachers' survival. Without the ability to learn, store and recall, teachers cannot face the presupposition of educational practice and reasonably face the dynamic practice. How can they deal with incidents? For example, the dynamically generated educational wit has deep memory.

\subsection{Optimizing the Efficient Procedural Memory}

Procedural memory is a memory route of "how to do". A lot of information in the procedural memory route is the processing of various processes made and remembered by the body. The storage of procedural memory allows teachers to do two or more things at the same time. Since these functions require the participation of two completely different regions in the brain, they do not conflict for the sake of brain space and energy, and the brain can easily allocate attention [13]. For example, the teachers take notes while listening to the trainer's lectures and comparing the past experience. How to make teachers optimize their own procedural strategies? One is to make teachers improve their skills in practical exercises through training until a program is formed, because when a program is often repeated, the brain will store it in the cerebellum for easy invocation; the other is to design some programs that are helpful for memory in training. In addition, everything that includes sports can strengthen procedural memory, so trainers and teachers can try a variety of sports, such as role-playing, debating, presentation, lectures, etc..

\subsection{Stimulating the Associative Automatic Memory}

The "associative" memory route refers to the automatic stimulation of memory or information by specific stimuli [14]. If a teacher hears a new educational theory from a trainer, and you happen to know the new theory, you can make a rough judgment about what will be said and even what will happen. Automatic memory can lead to the opening of other memory routes, which means when one memory route is activated, other memory routes may also be activated. The premise of automatic memory is that new concepts are combined or compared with known concepts, and unfamiliar information is linked to familiar information. If teachers need to learn new skills and new information, their desire for training can make teachers enthusiastic about participating in training. Trainers need to investigate what drives teacher training and what teachers want in order to establish a link between the training teachers need and what teachers want and make automatic memory play a greater role.

\subsection{Mobilizing the Positive Emotional Memory}

Human beings are emotional animals. Emotional information is always processed prior to other information, and the brain always gives the greatest priority to emotion. Positive feelings such as joy, happiness and cooperation with others have a strong impact on training, and strong emotions (both positive and negative emotions) are usually well remembered [15]. Hence, the brain first pays attention to the information with strong emotional content in the physiological program, and the time to remember the information in the program will be longer. When emotional memory controls the brain, logical ability will be lost. Emotional memory is the most efficient type of memory, and emotional memory can also activate other memory storage areas. However, for many years, perceptual thinking has been the center of attention, while emotion has been ignored. The research on brain has shown that emotion and thinking are closely related. Whether emotional quotient can be developed and whether emotion and thinking can be coordinated is one of the key factors for improving the effect of teacher training.

In order to effectively develop teachers' emotional memory, firstly, the trainers should show their enthusiasm for the training content and show their love for the whole activity, which is very infectious for teachers. If the trainers are willing to share their feelings about the training content, the trainee will find that he or she can also experience this feeling; secondly, the relaxed training environment should be created. In the relaxed training environment, teachers can respond and think analytically, but feel helpless and tired when they are in the highly stressed environment. Therefore, the relaxed and comfortable environment is conducive to developing people's potential and improving performance; thirdly, the establishment of confidence is also an important 
prerequisite for emotional memory. It can consciously help teachers improve self-confidence and self-esteem, build self-confidence and effectively activate multiple memory routes in combination with action research, studying and thinking about curriculum and other specific activities. In short, the ideal state is to be able to flexibly use five memory route strategies, which can simplify the work of trainers and get twice the result with half the effort. If the individuals are more familiar with the information, they are more likely to use it consciously. Teachers not only listen to lectures, but also take the initiative to participate in the training. Presentation and lecture are an effective way to use multiple routes, and the brain processes part and whole information at the same time. In addition to semantic information, emotional memory is also stimulated by the process and plot of lectures, whereas episodic memory is strengthened by the place and way of lectures.

\section{TRAINING THE CONTROL MODEL TO ACHIEVE THE SMOOTH MEMORY RETRIEVAL}

Frequently using the information stored in short-term memory allows nerves to establish stronger connections, namely stronger memories, and accurately lay the foundation through behavior. An important aspect of behavior is to be able to use one's brain to make decisions. In the teachers' educational practice, the educational behavioral decisions may be made due to extremely impulsive and insufficient thinking or the other extreme, which is extremely careful consideration, spending too much time considering all possible options and vacillation, leading to inefficient decisions, poor efficient decisions and losing opportunity. Among them, teachers' ability of memory retrieval and dispersion of effect affect the on-site performance of individual skills in a wide range. Teacher training strategies of activation memory are shown in Figure 1.

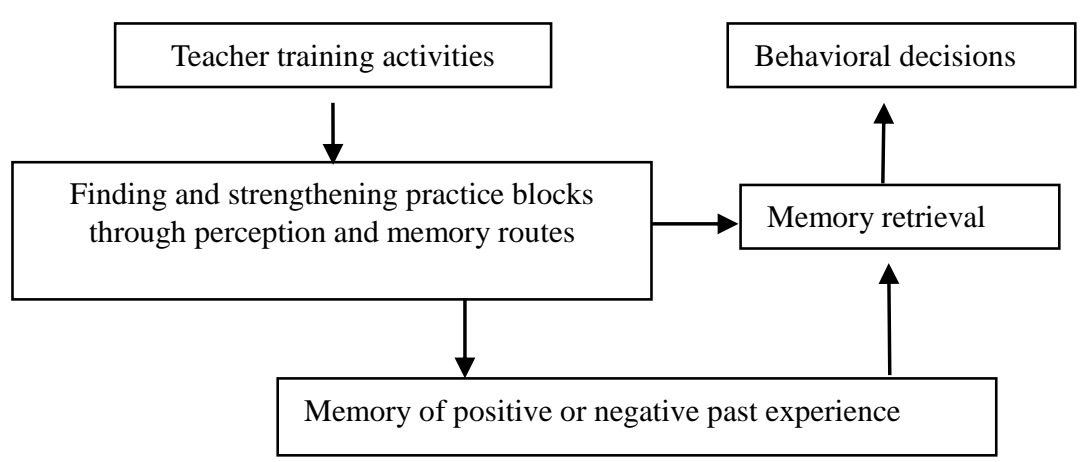

Figure 1 The control model of teacher training strategies for activation memory

Teacher training is an information processing activity that includes the storage and retrieval of information. If the memory information cannot be retrieved, it is a major deficiency of cognitive skill development. Sometimes, the memory cannot be retrieved, and the desired information cannot be obtained. It is not because the memory is not good, and it may be that the wrong route is used. In the training, teachers do not pay equal attention to any information in the perceptual range. However, they often pay special attention to particularly significant action skills in the perceptual range, especially in situations. This is the selection of information. Therefore, in order to guide the selection and memory of teachers in the training, it is necessary to provide some objective conditions to make some information "protrude" from other information and attract teachers' greatest attention, so as to absorb it into the cognitive system in the brain and carry out the effective memory retrieval. In order to promote teachers' smooth cognitive processing in the training process, teachers should regard themselves as students to inject emotion, believe in their cognitive skills and select the most handy strategies, and for example, actions, sounds, dialogues, music and storytelling can be adopted separately and together, which will improve the retrieval effect and performance. Moreover, teachers can also use mechanical repetition strategies and fine repetition strategies to promote memory retrieval. Theories of some practical skills (such as spoken expression) may be helpful, but require multiple repeated practices, namely the mechanical repetition strategies, so that individual teacher can use skills quickly and accurately while consciously performing other brain functions. Fine repetition strategies, including many strategies, can make information more meaningful or relevant for teachers, so as to enhance memory.

Teachers' memory and application of practical skills are limited by various factors, and this limitation can be remedied through "practice blocks". The practice block, the area of the teacher's cerebral cortex responsible for practical memory, controls most of the body's skills and is the reticular activation system that keeps the brain awake. There are differences in the performance of teachers' "practice blocks" in different professional development stages. The difference between novice teachers and expert teachers is that expert teachers tend to use larger blocks 
(due to a lot of practical experience) to integrate various skills and organize information, so the relevant information retrieval is accurate and efficient. However, novice teachers use isolated and small block practice to deal with information, which affects the retrieval of practical skills.

\section{CONCLUSION}

Overall, our study has analysed relationships between brain memory mechanism and effectiveness of teacher training, has studied the role of activation of five memory routes, namely semantic memory, episodic memory, procedural memory, automatic memory and emotional memory, in improving the effect of teacher training, and constructs the control model of teacher training strategies that activate memory. Through the control model and the flexible use of five memory route strategies, trainees' memory ability and memory extraction ability of information can be enhanced. Finally, the improvement of teacher training quality can be reflected in the teacher's behavioural decision-making.

\section{ACKNOWLEDGMENT}

This research work was financially supported by "Liupanshui Science and Technology Information Service Cloud Platform" of the Provincial-Municipal Co-construction Project of Science and Technology Department of Guizhou Province (52020-2016-04-05); "Liupanshui Science and Technology Information Service Cloud Platform" of the Fund Project of Science and Technology Bureau of Liupanshui (52020-2017-02-01); The Key Cultivation Discipline in Liupanshui Normal University (LPSSYZDPYXK201710); The Innovation Team of Liupanshui Normal University (LPSSYKJTD201502).

\section{REFERENCES}

[1] Z. M. Liu, C. Y. Guo, L. Luo, Attention and available long-term memory in an activation-based model, J. Science China Life Sciences, 53 (2010) 743-752.

[2] C. Ranganath, R. S. Blumenfeld, Doubts about double dissociations between short-and long-term memory, J. Trends Cogn Sci, 9 (2005) 374-380.

[3] H. Koizumi, Brain Science and

Education-Cutting-edge Research and Future Prospects, J. Educational Research, 2 (2006) 22-27.
[4] Z. Y. Jia, T. L. Xiao, Hideaki Koizumu's Discussion on Brain Science and Education, J. Reference for Basic Education, 12 (2010) 7-11+18.

[5] P.Wolfe, Functions of the Brain, translated by Center for Brain Science and Educational Application Research, China Light Industry Press, Beijing, 2005.

[6] J. C. Mao, Teachers' misinterpretation of Chinese Characters and Its Correction in Literacy Teaching, J. Modern Primary and Secondary Education, 31 (2015) 49-53.

[7] M. Sprenger, Learning and Memory of the Brain, translated by Center for Brain Science and Educational Application Research, China Light Industry Press, Beijing, 2005.

[8] N. Cowan, An embedded-process model of working memory, in: A. Miyake, P. Shah, (Eds.), Models of Working Memory: mechanis ms of Active Maintenance and Executive, Cambridge University Press, Cambridge, 1999, pp. 62-101.

[9] K. Oberauer, Access to information in working memory: exploring the focus of attention, J. Exp Psychol Learn Mem Cogn, 28 (2002) 411- 421.

[10] K. Oberauer. Is the focus of attention in working memory expanded through practice?, J. Exp Psychol Learn Mem Cogn, 32 (2006) 197-214.

[11] S. L. He, Discussion on Improving Students' Spiritual Life Quality by Teaching, Education Theory and Practice, 30 (2010) 61-64.

[12] R. Riding, S. Rainer, Cognitive Style and Learning Strategy, translated by W. G. Pang, East China Normal University Press, Shanghai, 2003.

[13] C. Zhang, Study and Application of Decision Network Model Based on Brain Neuron Sequence Memory, Nanjing University of Information Science \& Technology, 2018.

[14] D. M. Xiu, H. L. Xue. Research Progress on Neural Mechanisms of Learning and Memory, J. Bulletin of Biology, 48 (2013) 1-3.

[15] S. S. Rostrand, Accelerating Performance Improvement, translated by M. Y. Yang, Economic Management Press, Beijing, 2005. 